\title{
Advances in cancer modeling: fluidic systems for increasing representativeness of large 3D multicellular spheroids
}

\author{
Filippo Piccinini' ${ }^{1}$ Ilaria De Santis ${ }^{2,3}$ \& Alessandro Bevilacqua*,2,4
}

\section{"The HDN extends tradi- tional hanging- drop technology by creating a reconfigurable microfluidic network."}

\section{KEYWORDS:}

3D cell cultures $\cdot$ microfluidic systems - multicellular spheroid • preclinical studies • tumor microenvironment

'Istituto Scientifico Romagnolo per lo Studio e la Cura dei Tumori (IRST) IRCCS, Meldola (FC), Italy; ${ }^{2}$ Advanced Research Centre on Electronic Systems for Information \& Communication Technologies ' $E$. De Castro' (ARCES), University of Bologna, Italy; '3nterdepartmental Centre 'L. Galvani' for integrated studies of bioinformatics, biophysics \& biocomplexity (CIG), University of Bologna, Italy; ${ }^{4}$ Department of Computer Science \& Engineering (DISI), University of Bologna, Italy; *Author for correspondence: alessandro.bevilacqua@unibo.it
The representativeness of a cellular model is fundamental in preclinical cancer studies, being defined as the degree of likelihood between the in vitro model and the in vivo mimicked tumor situation. Although being the gold standard in cell biology for more than a half century, 2D cell cultures poorly represent the complex three-dimensionality of in vivo conditions. Size, heterogeneity and perfusion are three key aspects characterizing the behavior of the tumor and driving its progression. In this regard, it has been widely proven that 3D multicellular models enable a more reliable assessment of anti-cancer drugs and radiotherapy treatments, although a recent article emphasized that today there are no well-established approaches to obtain relevant biological data from these models [1]. Moreover, there are not even shared definitions of multicellular aggregates, spheroids, microtissues and organoids. Therefore, this work provides an opening rationale for the different terms today used for $3 \mathrm{D}$ cell cultures and discusses the benefits of using large-sized spheroids as 3D preclinical culture models. Finally, this work outlines the microfluidic systems that are now ready for 3D culture cultivation and manipulation in dynamic controlled conditions, this representing a further step towards more representative $3 \mathrm{D}$ in vitro cancer models.

Based on the structural and consequently functional complexity, multicellular aggregates can be considered as the simplest 3D cell cultures, as they are characterized by a 3D cell-cell aggregation free from a defined structural obligation. The commonly used term spheroids should be referred to those multicellular aggregates producing their own extracellular matrix and having a nearly spherical shape [2]. When spheroids comprise more than one cell types that together accomplish a specific function, referring to them as microtissues is more appropriate. However, the use of the terms spheroids and microtissues is still often overlapping. It is worth noting that if the spheroids are composed of more than one cell type not accomplishing a specific function together, they can be better identified as co-culture spheroids. Finally, self-renewing multicellular aggregates that self-organize into ex vivo mini-organs are named organoids [3].

Whilst complex cell culture models enable representation of many in vivo parameters and interactions, spheroids are a simple yet effective model to represent in vivo tumor conditions while retaining handiness in their generation and use. Furthermore, it is known that when their diameter exceeds the threshold of approximately $500 \mu \mathrm{m}$, spheroids become more heterogeneous, since they develop a necrotic core surrounded by a viable rim of quiescent cells and an outer layer of proliferating cells. This structure mimics the one of vascular tumor nodules and microregions of solid tumors, where cells next to capillaries are actively proliferating and distant inner cells stay quiescent or die due to mass transport limitations. Accordingly, stratified large-sized spheroids are a more representative model of in vivo intra-tumor heterogeneity, an aspect widely reported to impact on many cancer-specific traits such as clonogenicity, invasive potential and response to drugs [2].

A further improvement in the representativeness of a cellular model can be achieved by taking into account the tumor microenvironment, which has been extensively proven to play a key role in several steps of tumor progression, from initial neoplastic transformation to an eventual metastatic invasion. Traditional culture methods are static and thus often limiting for reshaping 
Dynamic in vivo conditions, where cells may undergo phenomena such as interstitial flow-derived mechanical stresses and spatial and temporal variation in chemical and physical factors. In this regard, microfluidic systems can be used as tools to actively mimic and modulate parameters of the culture environment [4].

Microfluidic systems are those platforms and technologies that allow manipulaton of tiny amounts of fluids using microstructures from tens to hundreds of micrometers. Microfluidic devices can be coupled to 3D cellular models to: (a) provide continuous perfusion of fresh culture medium, which replenishes cells with nutrients while removing toxic wastes; (b) perform a fine manipulation of biophysical and biochemical parameters, such as chemical gradients, metabolic stress and fluid flows; (c) allow real-time imaging and monitoring of the culture in dynamics, also through the integration of different biosensors on-board [5].

Many of the developed microfluidic devices permit high-throughput cultivation and parallel analysis of $3 D$ cell cultures. Nonetheless, there are still limits to their widespread use as general purpose systems. Precisely: (a) most devices are conceived just for microscale cultures and do not support 3D aggregates above hundreds of micrometers in size; (b) not all the existing microfluidic systems permit the individual interrogation of aggregates on the same device, nor their harvesting for later analysis [2]; (c) to support and protect the culture integrity, microfluidic devices often require the use of artificial scaffolds, which may have non-physiological compositions, leading to cellular aberrations [2]; (d) many microfluidic devices are of specific purpose, having complex peculiar capillary structure that results in a high tissue specificity, but also reduced applicability of the system out of its native context [6]. These drawbacks make common microfluidic systems unsuitable to culture in vitro 3D multicellular spheroids of relevant size (e.g., above $500 \mu \mathrm{m})$. In particular, only three technologies bypass these four drawbacks and are available at the present time: (a) the organon-chip device, developed by Schimek et al. (TissUse GmbH, Berlin, Germany) [7]; (b) the microfluidic hanging-drop network (HDN) by Misun et al. (InSphero AG, Schlieren,
Switzerland) [8]; (c) the multicompartmental modular bioreactor (MCmB) by Mazzei et al. (commercialized by Kirkstall Ltd, Rotherdam, UK and recently by IVTech Srl, Massarosa, Italy) [9]. A schematic of the culture unit of each of these technologies is reported in Supplementary Figure S1, whilst a comparative overview can be found in Supplementary Table S1.

The organ-on-chip device is a chip-sized system originally intended to investigate flow-driven shear stress effects on human endothelial cells. The system is endowed with an on-board micropump supporting steady long-term fluid flow through a microchannel system fully covered by cells [7]. Although initially developed as a tissuespecific platform, the system has been revised and is actually commercialized in the form of 2-organ-chip and 4-organchip by TissUse $\mathrm{GmbH}$. These systems respectively comprise two and four culture chamber units, thus enabling simultaneous cultivation of up to two and four different multicellular aggregates in a common media perfusion circuit at a miniaturized scale. The systems are also compatible with standard Transwell inserts and with matrix supports, thus allowing modelling biological barriers and the reproduction of parenchymal organs environment.

The HDN extends traditional hangingdrop technology by creating a reconfigurable microfluidic network. The device is based on a micropatterned surface of channels and circular areas for drop formation, resulting in a network of drops connected by a common flowing medium driven by an external pump. The medium perfusion permits substance dosage and nutrient supply, thus extending the in-culture maintenance of the spheroids. Moreover, capillary valves within the network enable the reconfiguration of drop interconnections and consequent different multi-aggregates experimental settings [8]. The system has recently been commercialized by InSphero AG under the name of 3D InSight"' Microtissues, namely an assay-ready system consisting of HCN-derived Akura"' plates, in 96- or 384-well format, which already incorporates preformed microtissues in different formats (InSphero AG).

$\mathrm{MCmB}$ is a modular fluidic system powered by an external peristaltic pump and equipped with a mixing chamber primarily acting as a gas exchanger and flow rectifier. The culture chamber's roof is specifically designed to minimize shear stress on the cells while applying relatively high flow rates that maximize oxygen delivery to the culture. Having the size of a 24-plate well, the chambers support routine laboratory protocols and procedures, including standard Transwell utilization to mimic epithelial structures. The MCmB is today available in several configurations (Supplementary Figure S2): (a) the QV500 Quasi Vivo chamber (Kirkstall Ltd), characterized by single inlet and outlet and a sloping roof; (b) the LiveBox1 (IVTech Srl), characterized by single inlet and outlet and a flat optically transparent roof for optimizing live optical imaging during the experiments; (c) the LiveBox2 (IVTech Srl), characterized by two inlets and two outlets, a flat optically transparent roof, and a membrane holder for physiological barrier simulation. In spite of their differences, all these system designs are based on allometric scaling principles, thus allowing for interconnection of different 2D/3D culture types in a metabolically and physiologically relevant manner [10].

In conclusion, this work presents the perfusion systems that are actually able to perform in vitro cultivation and manipulation of multicellular spheroids of relevant size in controlled conditions. We discussed

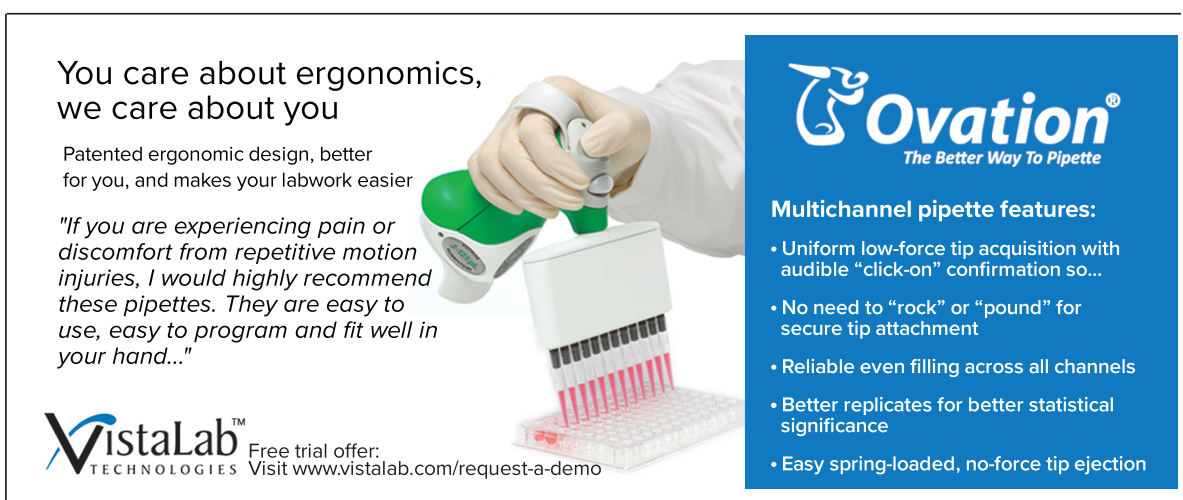


Dhow the adoption of such microfluidic systems for culture and interrogation of heterogeneous large-sized spheroids maximizes the in vitro resemblance of the in vivo complexity. Generation technologies and imaging systems are today ready to deal with large-size spheroids [1]. Herewith, if on one hand some advancements lack for extracting quantitative data at singlecell level and performing deep high-content screening analyses, on the other hand the use of fluidic systems together with spheroids larger than $500 \mu \mathrm{m}$ actually represents the best trade-off between handiness of a model's parameters and possibility to obtain reliable and reproducible biological data in preclinical studies.

\section{AUTHOR CONTRIBUTIONS}

$\mathrm{FP}$ and $\mathrm{AB}$ conceived and initiated the project. FP and IDS prepared and collected the data. FP, IDS wrote the manuscript. AB revised the manuscript. All authors read and approved the final manuscript.

\section{ACKNOWLEDGMENTS}

We would like to thank Kirkstall Ltd (Rotherham, UK) and IVTech Srl (Massarosa, Italy) for providing us with information about their systems, which we used to derive the schematics reported in the Supplementary data section.

\section{FINANCIAL DISCLOSURE}

The authors have no relevant affiliations or financial involvement with any organization or entity with a financial interest in or financial conflict with the subject matter or materials discussed in the manuscript. This includes employment, consultancies, honoraria, stock ownership or options, expert testimony, grants or patents received or pending, or royalties.

No writing assistance was utilized in the production of this manuscript.

\section{OPEN ACCESS}

This work is licensed under the AttributionNonCommercial-NoDerivatives 4.0 Unported License. To view a copy of this license, visit http://creativecommons.org/licenses/ by-nc-nd/4.0/

\section{SUPPLEMENTARY DATA}

To view the supplementary data that accompany this paper please visit the journal website at: www.future-science. com/doi/suppl/10.2144/btn-2018-0153

\section{REFERENCES}

1. Carragher N, Piccinini F, Tesei A, Trask JJ, Bickle M, Horvathet P. Concerns, challenges and promises of high-content analysis of 3D cellular models. Nat. Rev. Drug Discov. 17(8), 606 (2018).

2. Aijian AP, Garrell RL. Digital microfluidics for automated hanging drop cell spheroid culture. J. Lab. Autom. 20(3), 283-295 (2015).

3. Langhans SA. Three-dimensional in vitro cell culture models in drug discovery and drug repositioning. Front. Pharmacol. 9, (2018).

4. Hoh JH, Werbin JL, Heinz WF. Restricted exchange microenvironments for cell culture. BioTechniques 64(3), 101-109 (2018).

5. Vang S, Seitz K, Krysan PJ. A simple microfluidic device for live cell imaging of Arabidopsis cotyledons, leaves, and seedlings. BioTechniques 64(6), 255-261 (2018).

6. Young EWK, Simmons CA. Macro-and microscale fluid flow systems for endothelial cell biology. Lab Chip. 10(2), 143-160 (2010)

7. Schimek K, Busek M, Brincker S et al. Integrating biological vasculature into a multi-organ-chip microsystem. Lab Chip. 13(18), 3588-3598 (2013).

8. Misun PM, Birchler AK, Lang $M$ et al. Fabrication and operation of microfluidic hanging-drop networks. In: Cell-Based Microarrays. Humana Press, 183-202 (2018).

9. Mazzei D, Guzzardi MA, Giusti S et al. A low shear stress modular bioreactor for connected cell culture under high flow rates. Biotechnol. Bioeng. 106, 127-137 (2010).

10. Ahluwalia A. Allometric scaling in-vitro. Sci. Rep. 7 42113 (2017).

11. Frey 0 , Misun PM, Fluri DA et al. Reconfigurable microfluidic hanging drop network for multi-tissue interaction and analysis. Nat. Commun. 5, 4250 (2014). This reference has been cited in the Supplementary data section.

First draft submitted: 2 October 2018; Accepted for publication: 9 October 2018

\section{(1) \\ ONCOLOGY \\ Future Oncology}

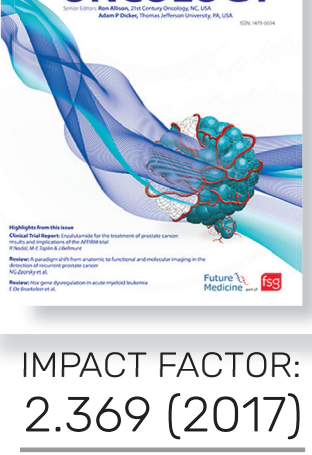

ISSN: 1479-6694

Frequency per year: 30

\section{INDEXING}

MEDLINE/Index Medicus, EMBASE/Excerpta Medica, Chemical Abstracts, $\mathrm{CINAHL}{ }^{\circledR}$ Plus, Science Citation Index Expanded ${ }^{\mathrm{TM}}$ (SciSearch ${ }^{\circledR}$ ), Journal Citation Reports/Science Edition ${ }^{\circledR}$, Current Contents ${ }^{\circledR} /$ Clinical Medicine, Scopus ${ }^{\circledR}$ 